\title{
Divergence of the Populations of Yellow Wagtail Motacilla flaval Linnaeus, 1758, And Citrine Wagtail Motacilla citreola Pallas, 1776, (Motacillidae, Passeriformes) in Middle Volga Region of Russia
}

\author{
E.A. Artemieva ${ }^{1}$, A.V. Mishchenko ${ }^{1} \&$ D.K. Makarov ${ }^{1}$ \\ ${ }^{1}$ I.N. Ulianov Ulianovsk State Teacher's Training University, the Centenary of V.I. Lenin's Birth sq., 4, Ulianovsk, \\ 432700, Russia \\ Correspondence: E.A. Artemieva, I.N. Ulianov Ulianovsk State Teacher's Training University, the Centenary of \\ V.I. Lenin's Birth sq., 4, Ulianovsk, 432700, Russia. E-mail: hart5590@gmail.com
}

Received: January 11, 2016 Accepted: February 20, 2016 Online Published: February 22, 2016

doi:10.5539/ijb.v8n2p32 URL: http://dx.doi.org/10.5539/ijb.v8n2p32

\begin{abstract}
Blood samples of so-called "yellow" wagtails collected in geographical areas of Middle Volga breeding populations of these species were studied. After mtDNA isolation barcoding of studied species of "yellow" wagtails was performed. Site of gene cytochromec-oxidase I was amplified. This gene was used as a genetic marker for the comparison of obtained samples. After sequencing and sequence alignment of gene cytochrome c-oxidase I, based on the comparison of genetic distances between specimens of the studied species using Jalview software, phylogenetic trees of populations of species Motacilla flava L. and Motacilla citreola Pall. were constructed.
\end{abstract}

Keywords: phenotype, genotype, mtDNA barkoding, population, species, birds, "yellow" wagtails, Middle Volga Region of Russia

\section{Introduction}

Among themost taxonomically controversial groups of passerinebirds polytypical complex Motacilla flava in sensu lato (Gladkov, 1954; Portenko, 1960; Stepanyan, 1990; Sotnikov, 2006; Artemieva \& Muraviev, 2012b) take saspecial place. An extremely complex individual and geographical variability is in herent to the forms of this group (Zarudny, 1891; Beregovoy, 1970; Bakhtadze, 1987; Grichik, 1992; Babenko, 1981; Red'kin, 2001a, 2001b; Muraviev, Artemieva, Beme, 2014; Cramp, 1988; Artemieva, Muraviev, \& Beme, 2013). In addition to the environmental and etological factors of reproductive isolation of sympatric bird sspecies molecular-genetic features of species can play an insulating role too.To identify the real kinship within the taxa an integrated approach that combines assessment of the variability of phenotypic and genotypic features of specific forms including molecular-genetic attributes is required (Artemieva \& Muraviev, 2012a; Pavlova et al., 2003; Vili et al., 2009).

Objective: To identify phenotypic and genotypic divergence in populations of yellow wagtail Motacilla flava and citrine wagtail Motacilla citreola under sympatric conditions in the Middle Volga region of Russia.

\section{Materials and Methods}

Species of the "yellow" wagtails Motacilla flava Linnaeus, 1758, and Motacilla citreola Pallas, 1776 (Passeriformes, Motacillidae) subgenus Budytes Cuiv. 1817, were selected as the object of molecular-genetic research. We used 11 dried blood samples of $M$. flava on filter paper (6-9.05.2012) and 9 dried blood samples of M. citreola on filter paper (5-9.05.2012) collected in the Nizhniy Novgorod (floodplains of Volga and Oka, wastewater treatment plants, meadows).

Samples for genetic studies were collecte dusing the non-invasive sampling method - the birds for blood sample picking were caught with arachnoid ornithological net. Morphometric and plumage characteristics were taken and the bird was ringed. Original value scale for gradation of morphometry parameters as well as plumage color and pattern of birds was developed (Table 1 \& 2). System of color and plumage pattern features by Ryabitsev (2001) was applied.

Blood samples were taken from the brachial vein of bird swhen ringing using IsoCode STIXTM paper (special impregnated paper that can be easily used in the field conditions. It significantly reduces the stress effects, as only 1-2 drops equal to 10-12 $\mu 1$ - an amount sufficient to genetic expertise - is taken). 
DNA isolation from dried blood samples was carried outon the paper (CosmoBio, Schleicher \& Schuell Biosciences) using GeneJET Whole Blood Genomic DNA Purification Mini Kit (Thermo Scientific). Samples were cut out from paper, homogenized and incubated in lysing solution (Lysis Solution, Thermo Scientific) containing proteinase $\mathrm{K}\left(56^{\circ} \mathrm{C}, 15\right.$ minutes). Further, the DNA was precipitated with $96 \%$ ethanol and recovered on silicon columns (GeneJET Genomic DNA Purification Columns, Thermo Scientific).

Fragment of the gene cytochrome $\mathrm{C}$ oxidase I (COI) was used as the genetic marker. To amplify the area of interest the following PCR mixture was used (for $20 \mu \mathrm{l})$ : dNTP $(250 \mu \mathrm{M})$, primers $(0.5 \mu \mathrm{M})$, the buffer (1X), taq-polymerase $(10 \mathrm{u})$, DNA template $(1 \mu \mathrm{l})$, deionized water (to a final volume). Polymerase chain reaction was performed using a thermal cycler FlexCycler (Analytik Jena) with the following temperature settings: DNA denaturation $-94^{\circ} \mathrm{C}, 2 \mathrm{~min}$; 30 cycles under the next conditions: DNA denaturation $-94^{\circ} \mathrm{C}, 30 \mathrm{sec}$, primer annealing $-55^{\circ} \mathrm{C}, 30 \mathrm{sec}$., elongation $-72^{\circ} \mathrm{C}, 40 \mathrm{sec}$.; chain completion $-72^{\circ} \mathrm{C}, 5 \mathrm{~min}$. The results of the reaction were assessed and the fragments were separated in $1 \%$ analytical agarose gel;after that preparative gel for the isolation and purification of the fragment of interest (using GeneJET Gel Extraction Kit (Thermo Scientific))was made.

The purified amplified products of the same length were sequenced using capillary genetic analyzer ABI PRISM 3500 (Life Technologies) (with preliminary sequencereaction with fluorescent-labeled deoxyribonucleotides (ddNTP) and subsequent purification of terminated fragment set). The obtained sequences were analyzed and adjusted using Sequence Scanner 2software (Life Technologies Corporation) [http://www.lifetechnologies.com]. The resulting sequence of the gene cytochrome $\mathrm{C}$ oxidase I was compared to the same available in GenBank [http://www.ncbi.nlm.nih.gov/genbank] and aligned using the ClustalW2 (EMBL-EBI) software. Genetic distances between specimens were determined using MEGA 4 software (Neighbor-joining method).

\section{Results}

Morphometry was carried out according to the standard procedures, the basic features of male and female plumage of yellow wagtail M. flava and citrine wagtail M. citreola were revealed, pairwise correlation analysis of plumage features and morphometry was performed. After that, the most informative features(uncorrelated with each other and with any one of the other parameters) were defined: for $M$. flava - tarsus length, body length, mustache coloration, for M. citreola - tarsus length, body length, necklace (Table 1-4).

Table 1. Morphometric parameters of Motacilla flava

\begin{tabular}{|c|c|c|c|c|c|c|c|c|c|c|c|c|}
\hline № & $\begin{array}{c}\text { Series,No. } \\
\text { of ring }\end{array}$ & $\begin{array}{l}\text { Catch } \\
\text { date }\end{array}$ & $\begin{array}{l}\text { Catch } \\
\text { time }\end{array}$ & $\begin{array}{l}\text { Sex } \\
(P 1)\end{array}$ & $\begin{array}{l}\text { Age } \\
\text { (P2) }\end{array}$ & $\begin{array}{l}\text { Fat } \\
\text { (P3) }\end{array}$ & $\begin{array}{c}\text { Weight } \\
\text { (P4) }\end{array}$ & $\begin{array}{c}\text { Wing } \\
\text { length } \\
\text { (P5) }\end{array}$ & $\begin{array}{c}\text { Tail } \\
\text { length } \\
\text { (P6) }\end{array}$ & $\begin{array}{c}\text { Tarsus } \\
\text { length } \\
\text { (P7) }\end{array}$ & $\begin{array}{l}\text { Cul1 } \\
\text { (P8) }\end{array}$ & $\begin{array}{l}\text { Body } \\
\text { length } \\
\text { (P9) }\end{array}$ \\
\hline $\mathrm{fl} 1$ & $\begin{array}{c}\mathrm{XH} \\
51036\end{array}$ & 6.05 & $18-20$ & $1 \mathrm{~m}$ & $1 \mathrm{ad}$ & 0 & 20,0 & 84,0 & 68,0 & 32,0 & 11,0 & 16,3 \\
\hline $\mathrm{fl} 2$ & $\begin{array}{c}\text { XH } \\
51039\end{array}$ & 6.05 & $19-45$ & $1 \mathrm{~m}$ & $1 \mathrm{ad}$ & 0 & 17,0 & 82,0 & 76,0 & 30,0 & 11,0 & 16,0 \\
\hline $\mathrm{fl} 3$ & $\begin{array}{c}\text { XH } \\
51046\end{array}$ & 7.05 & $9-05$ & $1 \mathrm{~m}$ & $1 \mathrm{ad}$ & 3 & 16,6 & 81,0 & 72,0 & 23,1 & 9,3 & 16,7 \\
\hline $\mathrm{fl} 4$ & $\begin{array}{c}\text { XH } \\
51048\end{array}$ & 7.05 & $10-30$ & $1 \mathrm{~m}$ & $1 \mathrm{ad}$ & 2 & 17,1 & 81,0 & 76,0 & 23,5 & 9,0 & 17,2 \\
\hline $\mathrm{fl} 5$ & $\begin{array}{c}\text { XH } \\
51051\end{array}$ & 7.05 & $20-40$ & $2 \mathrm{f}$ & $1 \mathrm{ad}$ & 0 & 15,0 & 75,0 & 71,0 & 23,43 & 10,0 & 14,5 \\
\hline fl6 & $\begin{array}{c}\text { XH } \\
51052\end{array}$ & 7.05 & $20-40$ & $1 \mathrm{~m}$ & $1 \mathrm{ad}$ & 0 & 18,5 & 83,0 & 75,0 & 24,91 & 11,0 & 16,1 \\
\hline $\mathrm{fl} 7$ & $\begin{array}{c}\text { XH } \\
51053\end{array}$ & 8.05 & $6-45$ & $1 \mathrm{~m}$ & $1 \mathrm{ad}$ & 0 & 16,5 & 82,0 & 71,0 & 23,3 & 9,7 & 16,5 \\
\hline $\mathrm{fl} 8$ & $\begin{array}{c}\text { XH } \\
51058\end{array}$ & 8.05 & $10-20$ & $1 \mathrm{~m}$ & $1 \mathrm{ad}$ & 1 & 17,3 & 86,0 & 74,0 & 23,0 & 9,5 & 16,3 \\
\hline $\mathrm{fl} 9$ & $\begin{array}{c}\text { XH } \\
51060\end{array}$ & 8.05 & $12-30$ & $1 \mathrm{~m}$ & $1 \mathrm{ad}$ & 0 & 16,7 & 83,0 & 71,0 & 24,9 & 9,3 & 15,4 \\
\hline fl10 & $\begin{array}{c}\text { XH } \\
51067\end{array}$ & 9.05 & $8-10$ & $1 \mathrm{~m}$ & $1 \mathrm{ad}$ & 1 & 17,5 & 84,0 & 68,0 & 23,8 & 9,5 & 15,5 \\
\hline fl11 & XH 51073 & 9.05 & $21-40$ & $2 \mathrm{f}$ & $1 \mathrm{ad}$ & 0 & 16,4 & 78,0 & 70,0 & 23,8 & 8,7 & 14,0 \\
\hline
\end{tabular}


Plumage pattern and color features of Motacilla flava expressed in points:

fl1, XH 51036 (series and number of the ring), catch date - 6.05, catch time - 18-20, sex (1 m), subspecies thunbergi, white whiskers (P10) -1 point, dark gray bregma (P11) - 2 points, narrow eyebrow (P12) - 1 point, white chin (P13) - 1 point, dark gray ear coverts (P14) - 2 points, olive-green back (P15) -2 points, bright yellow breast (P16) -3 points.

$>\mathrm{fl} 2$, XH 51039 (series and number of the ring), catch date- 6.05, catch time- 19-45, sex (1 m), subspecies thunbergi, white mustache (P10) - 1 point, dark gray bregma (P11) - 2 points, narrow eyebrow (P12) - 1 point, yellow chin $(\mathrm{P} 13)-3$ points, dark gray ear coverts $(\mathrm{P} 14)-2$ points, olive-green back $(\mathrm{P} 15)-2$ points, bright yellow breast (P16) -3 points.

$>\mathrm{fl}$, XH 51046 (series and number of the ring), catch date - 7.05, catch time - 9-05, sex (1 m), subspecies flava, white whiskers( $(\mathrm{P} 10)-1$ point, gray bregma (P11) - 1 point, wide eye brow (P12) -2 points, white chin $(\mathrm{P} 13)-1$ point, gray ear coverts $(\mathrm{P} 14)-1$ point, olive-green back(P15) -2 points, bright yellow breast (P16) -3 points.

$>\mathrm{fl} 4 \mathrm{XH} 51048$ (series and number of the ring), catch date- 7.05, catch time- 10-30, sex (1 m), subspecies flava, half white whiskers( $(\mathrm{P} 10)-2$ points, gray bregma (P11) - 1 point, wide eyebrow (P12) -2 points, white chin $(\mathrm{P} 13)-1$ point, gray ear coverts $(\mathrm{P} 14)-1$ point, olive-green back $(\mathrm{P} 15)-2$ points, bright yellow breast (P16) -3 points.

$>\mathrm{f15}, \mathrm{XH} 51051$ (series and number of the ring), catch date - 7.05, catch time - 20-40, sex (2 f), subspecies flava, grayish whiskers(P10) - 3 points, gray bregma (P11) - 1 point, wide eyebrow (P12) - 2 points, whitishyellowish chin $(\mathrm{P} 13)-2$ points, gray ear coverts $(\mathrm{P} 14)-1$ point, olive-grayish back(P15) - 1 point, grayishyellowish breast (P16) -1 point.

$>$ fl6, XH 51052 (series and number of the ring), catch date - 7.05, catch time - 20-40, sex (1 m), subspecies thunbergi, half white whiskers(P10) -2 points, dark gray bregma (P11) - 2 points, narrow eyebrow (P12) 1 point, yellow chin( $(\mathrm{P} 13)-3$ points, dark gray ear coverts $(\mathrm{P} 14)-2$ points, olive-green back(P15) -2 points, bright yellow breast $(\mathrm{P} 16)-3$ points.

$>\mathrm{fl}$, XH 51053 (series and number of the ring), catch date -8.05 , catch time $-6-45$, sex $(1 \mathrm{~m})$, subspecies flava, white whiskers(P10) - 1 point, gray bregma (P11) - 1 point, wide eyebrow (P12) - 2 points, white chin (P13) - 1 point, gray ear coverts $(\mathrm{P} 14)-1$ point, olive-green back(P15) - 2 points, yellow breast with dark spots (P16) -2 points.

$>\mathrm{fl} 18, \mathrm{XH} 51058$ (series and number of the ring), catch date -8.05 , catch time $-10-20$, sex $(1 \mathrm{~m})$, subspecies thunbergi, half white whiskers(P10) - 2 points, dark gray bregma (P11) - 2 points, no eyebrow (P12) - 0 points, white chin $(\mathrm{P} 13)-1$ point, dark gray ear coverts (P14) - 2 points, olive-green back( $\mathrm{P} 15)-2$ points, yellow breast with dark spots (P16) - 2 points.

$>\mathrm{fl}$, XH 51060 (series and number of the ring), catch date -8.05 , catch time $-12-30$, sex $(1 \mathrm{~m})$, subspecies thunbergi, yellow whiskers(P10) -4 points, dark gray bregma (P11) -2 points, narrow eyebrow $(\mathrm{P} 12)-1$ point, yellow chin $(\mathrm{P} 13)-3$ points, dark gray ear coverts $(\mathrm{P} 14)-2$ points, olive-green back $(\mathrm{P} 15)-2$ points, bright yellow breast ( $\mathrm{P} 16)-3$ points.

$>$ fl10, XH 51067 (series and number of the ring), catch date -9.05 , catch time $-8-10$, sex $(1 \mathrm{~m})$, subspecies flava, grayish-yellow whiskers(P10) - 5 points, dark gray bregma (P11) - 1 point, wide eyebrow (P12) - 2 points, yellow chin( $\mathrm{P} 13)-3$ points, gray ear coverts (P14) - 1 point, olive-green back(P15) -2 points, yellow breast with dark spots (P16) - 2 points.

> f111 (no blood sample), XH 51073 (series and number of the ring), catch time - 9.05, catch time - 21-40, sex(2 f), subspecies flava, grayish whiskers(P10) - 3 points, gray bregma $(\mathrm{P} 11)-1$ point, wide eyebrow (P12) - 2 points, whitish-yellowish chin (P13) - 2 points, gray ear coverts (P14) - 1 point, olive-grayish back(P15) - 1 point, grayish-yellowish breast(P16) - 1 point. 
Table 2. Morphometric parameters of Motacilla citreola

\begin{tabular}{|c|c|c|c|c|c|c|c|c|c|c|c|c|}
\hline No. & $\begin{array}{c}\text { Series and } \\
\text { number of the } \\
\text { ring }\end{array}$ & $\begin{array}{l}\text { Catch } \\
\text { date }\end{array}$ & $\begin{array}{l}\text { Catch } \\
\text { time }\end{array}$ & $\begin{array}{l}\text { Sex } \\
(\mathrm{P} 1)\end{array}$ & $\begin{array}{l}\text { Age } \\
(\mathrm{P} 2)\end{array}$ & $\begin{array}{l}\text { Fat } \\
(\mathrm{P} 3)\end{array}$ & $\begin{array}{c}\text { Weight } \\
\text { (P4) }\end{array}$ & $\begin{array}{l}\text { Wing } \\
\text { length } \\
\text { (P5) }\end{array}$ & $\begin{array}{c}\text { Tail } \\
\text { length } \\
\text { (P6) }\end{array}$ & $\begin{array}{l}\text { Tarsus } \\
\text { length } \\
\text { (P7) }\end{array}$ & $\begin{array}{l}\text { Cul1 } \\
\text { (P8) }\end{array}$ & $\begin{array}{l}\text { Body } \\
\text { length } \\
\text { (P9) }\end{array}$ \\
\hline ct1 & $\begin{array}{c}\text { XH } \\
47691\end{array}$ & 5.05 & $17-20$ & $1 \mathrm{~m}$ & $1 \mathrm{ad}$ & 0 & 25,0 & 80,0 & 65,0 & 27,0 & 11,0 & 15,0 \\
\hline ct2 & $\begin{array}{c}\text { XH } \\
51041\end{array}$ & 7.05 & $6-15$ & $2 \mathrm{f}$ & $1 \mathrm{ad}$ & 0 & 15,0 & 77,0 & 71,0 & 23,8 & 13,9 & 14,8 \\
\hline ct3 & $\begin{array}{c}\text { XH } \\
51042\end{array}$ & 7.05 & $6-15$ & $2 \mathrm{f}$ & $1 \mathrm{ad}$ & 0 & 16,0 & 79,0 & 72,5 & 24,5 & 9,9 & 16,0 \\
\hline ct 4 & $\begin{array}{c}\text { XH } \\
51043\end{array}$ & 7.05 & $6-15$ & $1 \mathrm{~m}$ & $1 \mathrm{ad}$ & 0 & 16,0 & 85,0 & 75,0 & 23,56 & 9,7 & 16,0 \\
\hline ct5 & $\begin{array}{c}\text { XH } \\
51044\end{array}$ & 7.05 & $6-15$ & $1 \mathrm{~m}$ & $1 \mathrm{ad}$ & 0 & 16,0 & 83,0 & 72,0 & 23,77 & 9,5 & 16,0 \\
\hline ct6 & $\begin{array}{c}\text { XH } \\
51045\end{array}$ & 7.05 & $9-05$ & $2 \mathrm{f}$ & $1 \mathrm{ad}$ & 0 & 16,75 & 79,0 & 69,0 & 23,16 & 10,4 & 15,5 \\
\hline ct7 & $\begin{array}{c}\text { XH } \\
51047\end{array}$ & 7.05 & $9-50$ & $1 \mathrm{~m}$ & $1 \mathrm{ad}$ & 1 & 16,1 & 79,0 & 72,0 & 24,6 & 9,7 & 16,0 \\
\hline ct8 & $\begin{array}{c}\text { XH } \\
51049\end{array}$ & 7.05 & $10-30$ & $1 \mathrm{~m}$ & $1 \mathrm{ad}$ & 1 & 15,6 & 82,0 & 71,0 & 24,3 & 9,9 & 15,2 \\
\hline ct 9 & $\begin{array}{c}\text { XH } \\
51068 \\
\text { hybrid }\end{array}$ & 9.05 & $8-10$ & $1 \mathrm{~m}$ & $1 \mathrm{ad}$ & 1 & 18,3 & 83,0 & 73,0 & 24,8 & 9,9 & 16,8 \\
\hline & $\begin{array}{c}\mathrm{XH} \\
50736\end{array}$ & & & & & & & & & & & \\
\hline ct10 & $\begin{array}{c}\text { Second } \\
\text { catch2011 г. } \\
\text { hybrid }\end{array}$ & 9.05 & $9-20$ & $2 \mathrm{f}$ & 0 & 1 & 17,9 & 76,0 & 65,0 & 24,0 & 10,1 & 14,9 \\
\hline
\end{tabular}

Plumage pattern and color features of Motacilla citreola expressed in points:

ct1, XH 47691 (series and number of the ring), catch date - 5.05, sex(2 f), subspecies citreola, no eyebrow (P10) - 0 points, black wide neck (nape) (P11) - 2 points, lemon yellow bregma (P12) - 4 points, dark gray back (P13) - 3 points, lemon yellow breast (P14) - 4 points, small black spot at the occiput (P15) - 3 points, wide strips on the wing $(\mathrm{P} 16)-2$ points, no necklace $(\mathrm{P} 17)-0$ points, lemon yellow throat (P18) -5 points.

ct2, XH 51041 (series and number of the ring), catch date - 7.05, sex (2 f), subspecies werae, yellowish eyebrow (P10) - 1 point, no neck (nape) (P11) - 0 points, pale yellowish bregma (P12) - 3 points, light gray back $(\mathrm{P} 13)-1$ point, dirty-gray breast $(\mathrm{P} 14)-1$ point, gray occiput $(\mathrm{P} 15)-1$ point, narrow strips on the wing $(\mathrm{P} 16)-1$ point, no necklace $(\mathrm{P} 17)-0$ points, yellowish throat $(\mathrm{P} 18)-1$ point.

ct3, XH 51042 (series and number of the ring), catch date - 7.05, sex (2 f), subspecies citreola, yellow eye brow $(\mathrm{P} 10)-2$ points, no neck (nape) $(\mathrm{P} 11)-0$ points, gray-yellow bregma $(\mathrm{P} 12)-2$ points, gray back $(\mathrm{P} 13)$ -2 points, yellowish breast $(\mathrm{P} 14)-2$ points, dark gray occiput $(\mathrm{P} 15)-2$ points, narrow strips on the wing (P16) - 1 point, necklace $(\mathrm{P} 17)-1$ point, ocher yellow throat $(\mathrm{P} 18)-3$ points.

$>\quad \mathrm{ct} 4, \mathrm{XH} 51043$ (series and number of the ring), catch date -7.05 , sex $(1 \mathrm{~m})$, subspecies werae, no eye brow (P10) - 0 points, narrow dark gray neck (nape) (P11) - 2 points, lemon yellow bregma (P12) - 4 points, gray back $(\mathrm{P} 13)-2$ points, pale lemon yellow breast $(\mathrm{P} 14)-3$ points, gray occiput $(\mathrm{P} 15)-1$ point, wide strips on the wing (P16) - 2 points, no necklace $(\mathrm{P} 17)-0$ points, lemon yellow throat (P18) -5 points.

$>\quad \mathrm{ct5}$, XH 51044 (series and number of the ring), catch date - 7.05, sex (1 m), subspecies citreola, no eye brow (P10) - 0 points, black wide neck (nape) (P11) - 2 points, lemon yellow bregma (P12) - 4 points, dark gray back $(\mathrm{P} 13)-3$ points, lemon yellow breast (P14) - 4 points, no black spot at the occiput $(\mathrm{P} 15)-5$ points, wide strips on the wing $(\mathrm{P} 16)-2$ points, necklace $(\mathrm{P} 17)-1$ point, lemon yellow throat $(\mathrm{P} 18)-5$ points.

$>\quad$ ct6, XH 51045 (series and number of the ring), catch date -7.05 , sex $(2 \mathrm{f})$, subspecies citreola, yellow eye brow (P10) - 2 points, no neck (nape) (P11) - 0 points, gray-yellow bregma (P12) - 3 points, light gray $\operatorname{back}(\mathrm{P} 13)-1$ point, yellowish breast $(\mathrm{P} 14)-2$ points, gray occiput $(\mathrm{P} 15)-1$ point, narrow strips on the wing $(\mathrm{P} 16)-1$ point, no necklace $(\mathrm{P} 17)-0$ points, yellow throat $(\mathrm{P} 18)-2$ points.

ct7, XH 51047 (series and number of the ring), catch date - 7.05, sex (1 m), subspecies werae, no eye brow $(\mathrm{P} 10)-0$ points, narrow dark gray neck (nape) $(\mathrm{P} 11)-2$ points, lemon yellow bregma (P12) - 4 points, gray 
back $(\mathrm{P} 13)-2$ points, pale lemon yellow breast $(\mathrm{P} 14)-3$ points, gray occiput $(\mathrm{P} 15)-1$ point, wide strips on the wing $(\mathrm{P} 16)-2$ points, no necklace $(\mathrm{P} 17)-0$ points, lemon yellow throat $(\mathrm{P} 18)-5$ points.

$>\quad$ ct8, XH 51049 (series and number of the ring), catch date- 7.05, sex (1 m), subspecies werae, no eyebrow (P10) - 0 points, narrow dark gray neck (nape) (P11) - 2 points, lemon yellow bregma (P12) - 4 points, gray back $(\mathrm{P} 13)-2$ points, lemon yellow breast $(\mathrm{P} 14)-4$ points, big black spot at the occiput $(\mathrm{P} 15)-4$ points, wide strips on the wing $(\mathrm{P} 16)-2$ points, no necklace $(\mathrm{P} 17)-0$ points, lemon yellow throat $(\mathrm{P} 18)-5$ points.

$>\quad$ ct9, ХН 51068 (series and number of the ring), гибрид, catch date- 9.05, sex (1 m), subspecies werae, no eye brow (P10) - 0 points, narrow dark gray neck (nape) (P11) - 2 points, lemon yellow bregma(P12) - 4 points, gray back $(\mathrm{P} 13)-2$ points, lemon yellow breast $(\mathrm{P} 14)-4$ points, big black spot at the occiput $(\mathrm{P} 15)$ -4 points, wide strips on the wing $(\mathrm{P} 16)-2$ points, no necklace $(\mathrm{P} 17)-0$ points, lemon yellow throat $(\mathrm{P} 18)$ -5 points.

$>\quad \operatorname{ct} 10, \mathrm{XH} 51736$ (series and number of the ring), hybrid, second catch 2011 г., catch date- 9.05, $\operatorname{sex}(2 \mathrm{f})$, subspecies citreola, ocher-yellow eyebrow (P10) - 3 points, no neck (nape) (P11) - 0 points, gray bregma $(\mathrm{P} 12)-1$ point, gray back $(\mathrm{P} 13)-2$ points, yellowish breast $(\mathrm{P} 14)-2$ points, gray occiput $(\mathrm{P} 15)-1$ point, narrow strips on the wing $(\mathrm{P} 16)-1$ point, necklace $(\mathrm{P} 17)-1$ point, ocher yellowish throat $(\mathrm{P} 18)-4$ points.

Table 3. Correlations between morphometry and feather coloration characters of Motacilla flava. Marked characters and correlations are significant at $\mathrm{p}<0,05 ; \mathrm{N}=11$ (casewise deletion of missing data)

\begin{tabular}{|c|c|c|c|c|c|c|c|c|c|c|c|c|c|c|c|}
\hline Chat & P1 & P3 & $\mathrm{P} 4$ & P5 & P6 & P7 & P8 & P9 & P10 & P11 & P12 & P13 & P14 & P15 & P16 \\
\hline $\mathrm{P} 1$ & 000 & 306 & 561 & 851 & $-0,256$ & $-0,237$ & $-0,279$ & 840 & 267 &, 430 & 392 & 048 &, 430 & $-1,000$ & 0,800 \\
\hline P3 & 06 & 000 & 093 & 093 & 0,202 & $-0,397$ & $-0,449$ & 39 & 38 & 407 & 258 &, 450 &, 407 &, 306 & ,383 \\
\hline $\mathrm{r}=$ & 561 & 3 & 0 & 6 & 2 & 0, & 0 , & 0 & 47 & 67 & 48 & 54 & 7 & 61 & 56 \\
\hline P5 & 851 & 0,093 & 0,686 & 0 & 157 & 248 & 0,224 & 72 & 78 & 591 & 653 & $-0,010$ &, 591 & 351 & ,537 \\
\hline $\mathrm{P}$ & 256 & 202 & 122 & 57 & 1,000 & $-0,088$ & 0 & & & 64 & ,301 & 0,037 & 64 & 56 & ,263 \\
\hline r t & 1 & 397 & 0,644 & $\mathrm{FO}_{\mathrm{O}}$ & $-0,088$ & 1,0 & ,739 & & -0 & 98 & 5 & 2 &, 598 & 37 & 34 \\
\hline 2 & & 4 & 0,540 & 0,224 & 0,150 & 0,739 & 0 & & & 5 & & & 25 & 79 & 21 \\
\hline & & & & & & & & & & & & & & & \\
\hline & & $-0,138$ & & & & & & & & & & & & & \\
\hline & 430 & , & & & 0 & & & & & & & & & & ולד, \\
\hline & 92 & 0,20 & $-0,448$ & $-0,653$ & $-0,301$ & -0, & $-0,419$ & & & & 1,000 & $-0,084$ & & $-0,392$ & $-0,25$ \\
\hline & 948 & $-0,450$ & $-0,054$ & $-0,010$ & 0,037 & 01 & 0,270 & -0 , & 0 , & 95 & $-0,084$ & 1,000 & 0,295 & $-0,048$ & $-0,098$ \\
\hline P14 & 430 & ,407 & 0,567 & 0,591 & 0264 & 05 & 0,625 & 8 & $-0,194$ & 1,000 & $-0,911$ & 0,295 & 1,000 & 430 & 0,399 \\
\hline & $-1,000$ & 306 & 0 & & 6 & & & & & & $-0,392$ & $-0,048$ & 30 & 00 & 00 \\
\hline P1 & 0,800 & 383 & 0,456 & 0,537 & 0,263 & 0,464 & 0,221 & $\mathbf{0 , 7 3 8}$ & $-0,360$ & 0,399 & $-0,252$ & $-0,098$ & 0,399 & $\mathbf{0 , 8 0 0}$ & 1,000 \\
\hline
\end{tabular}

Table 4. Correlations between morphometry and feather coloration characters of Motacilla citreola. Marked * correlations are significant at $\mathrm{p}<0,01 ; \mathrm{N}=10$ (casewise deletion of missing data)

\begin{tabular}{|c|c|c|c|c|c|c|c|c|c|c|c|c|c|c|c|c|c|c|}
\hline Characters & P1 & P2 & P3 & P4 & P5 & P6 & P7 & P8 & P9 & P10 & P11 & P12 & $\mathrm{P} 13$ & P14 & P15 & P16 & P17 & P18 \\
\hline P2 & $-0,408$ & 1,000 & $-0,408$ & $-0,077$ & 0,526 & 0,590 & 0,115 & 0,081 & 0,392 & $-0,680$ & 408 & 0,680 & 000 & 87 & 0,291 & 408 & $-0,509$ & $-0,000$ \\
\hline P3 & $-0,250$ & $-0,408$ & 1,000 & $-0,086$ & $-0,090$ & $-0,078$ & 0,061 & $-0,330$ & 0,140 & $-0,037$ & 0,250 & 0,037 & 0,000 & 0,273 & 0,109 & 0,250 & $-0,089$ & 0,433 \\
\hline P4 & $-0,253$ & $-0,077$ & $-0,086$ & 1,000 & $-0,042$ & $-0,655$ & 0,865 & 0,009 & $-0,196$ & $-0,132$ & 0,253 & 0,132 & 0,531 & 0,401 & 0,177 & 253 & $-0,150$ & 0,321 \\
\hline P5 & $-0,764$ & 0,526 & $-0,090$ & 1,000 & 1,000 & 0,631 & $-0,021$ & $-0,499$ & 0,627 & $-0,729$ & 0,764 & 0,729 & 0,406 & 0,714 & 0,546 & 0,764 & $-0,232$ & 0,623 \\
\hline P6 & $-0,306$ & 0,590 & $-0,078$ & 0,631 & 0,631 & 1,000 & $-0,437$ & $-0,205$ & 0,704 & $-0,456$ & 0,306 & 0,456 & $-0,151$ & 0,093 & 0,114 & 0,306 & $-0,149$ & 0,101 \\
\hline P7 & $-0,392$ & 0,115 & 0,061 & $-0,021$ & $-0,021$ & $-0,437$ & 1,000 & 0,031 & $-0,099$ & $-0,339$ & 0,392 & 0,339 & 0,599 & 0,472 & 0,290 & 0,392 & $-0,168$ & 0,408 \\
\hline P8 & 0,446 & 0,081 & $-0,330$ & $-0,499$ & $-0,499$ & $-0,205$ & 0,031 & 1,000 & $-0,592$ & 0,120 & $-0,446$ & $-0,120$ & $-0,486$ & $-0,581$ & $-0,321$ & $-0,446$ & $-0,301$ & $-0,727$ \\
\hline P9 & $-0,427$ & 0,392 & 0,140 & 0,627 & 0,627 & 0,704 & $-0,099$ & $-0,592$ & 1,000 & $-0,373$ & 0,427 & 0,373 & 0,181 & 0,410 & 0,323 & 0,427 & 0,014 & 0,416 \\
\hline P10 & 0,909 & $-0,680$ & $-0,037$ & $-0,729$ & $-0,729$ & $-0,456$ & $-0,339$ & 0,120 & $-0,373$ & 1,000 & $-0,909$ & $-1,000$ & $-0,440$ & $-0,729$ & $-0,524$ & $-0,909$ & 0,526 & $-0,590$ \\
\hline P11 & 1,000 & 0,408 & 0,250 & 0,764 & 0,764 & 0,306 & 0,392 & $-0,446$ & 0,427 & $-0,909$ & 1,000 & 0,909 & 0,645 & 0,899 & 0,576 & 1,000 & $-0,356$ & 0,866 \\
\hline P12 & $-0,909$ & 0,680 & 0,037 & 0,729 & 0,729 & 0,456 & 0,339 & $-0,120$ & 0,373 & $-1,000$ & 0,909 & 1,000 & 0,440 & 0,729 & 0,524 & $\mathbf{0 , 9 0 9}$ & $-0,526$ & 0,590 \\
\hline P13 & $-0,645$ & 0,000 & 0,000 & 0,406 & 0,406 & $-0,151$ & 0,599 & $-0,486$ & 0,181 & $-0,440$ & 0,645 & 0,440 & 1,000 & 0,757 & 0,638 & 0,645 & 0,345 & 0,782 \\
\hline P14 & $-0,899$ & 0,287 & 0,273 & 0,714 & 0,714 & 0,093 & 0,472 & $-0,581$ & 0,410 & $-0,729$ & 0,899 & 0,729 & 0,757 & 1,000 & 0,792 & 0,899 & $-0,146$ & 0,880 \\
\hline P15 & $-0,576$ & 0,291 & 0,109 & 0,546 & 0,546 & 0,114 & 0,290 & $-0,321$ & 0,323 & $-0,524$ & 0,576 & 0,524 & 0,638 & 0,792 & 1,000 & 0,576 & 0,161 & 0,523 \\
\hline P16 & 1,000 & 0,408 & 0,250 & 0,764 & 0,764 & 0,306 & 0,392 & $-0,446$ & 0,427 & $-0,909$ & 1,000 & 0,909 & 0,645 & 0,899 & 0,576 & 1,000 & $-0,356$ & 0,866 \\
\hline P17 & 0,356 & $-0,509$ & $-0,089$ & $-0,232$ & $-0,232$ & $-0,149$ & $-0,168$ & $-0,300$ & 0,014 & 0,526 & $-0,356$ & $-0,526$ & 0,345 & $-0,146$ & 0,161 & $-0,356$ & 1,000 & 0,000 \\
\hline P18 & $-0,866$ & 0,000 & 0,433 & 0,623 & 0,623 & 0,101 & 0,408 & $-0,727$ & 0,416 & $-0,590$ & 0,866 & 0,590 & 0,782 & $\mathbf{0 , 8 8 0}$ & 0,523 & 0,866 & 0,000 & 1,000 \\
\hline
\end{tabular}


On the base of the data obtained by the pairwise correlation analysis of morphometry and plumage features, the tree diagram of studied parameters was built using Ward cluster analysis and their clusters were allocated (Figure 1).

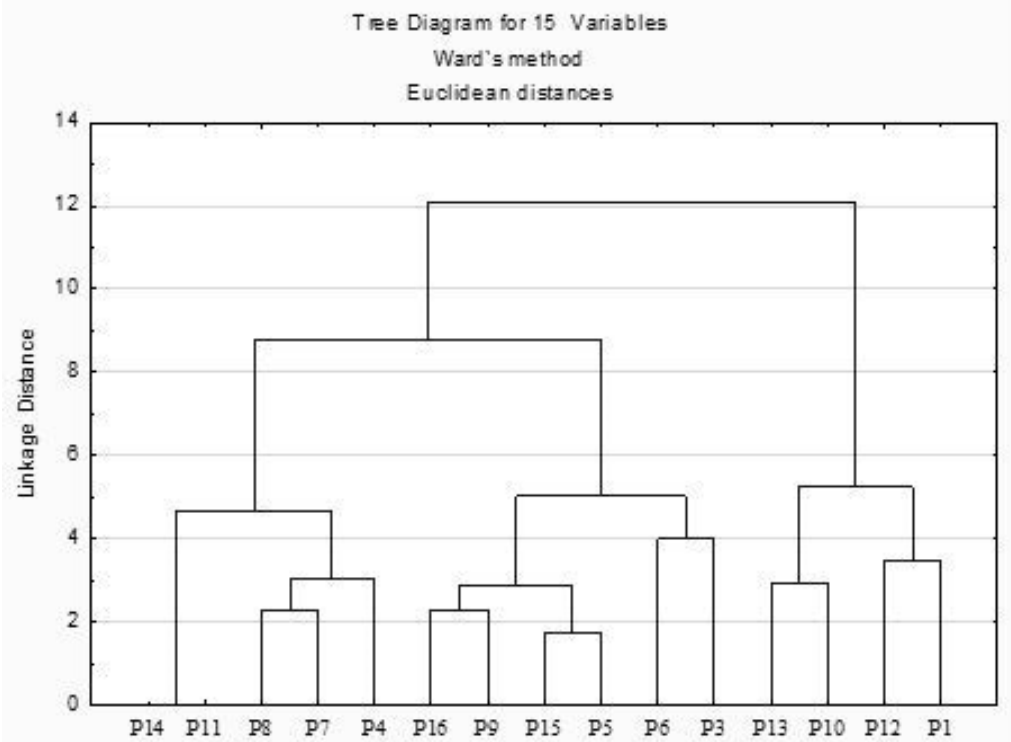

Figure 1. Clusters of morphometry and plumage parameters of Motacilla flava obtained with Ward method

On the base of the data obtained by the pairwise correlation analysis of morphometry and plumage features, the tree diagram of studied parameters was built using Ward cluster analysis and their clusters were allocated (Figure 2).

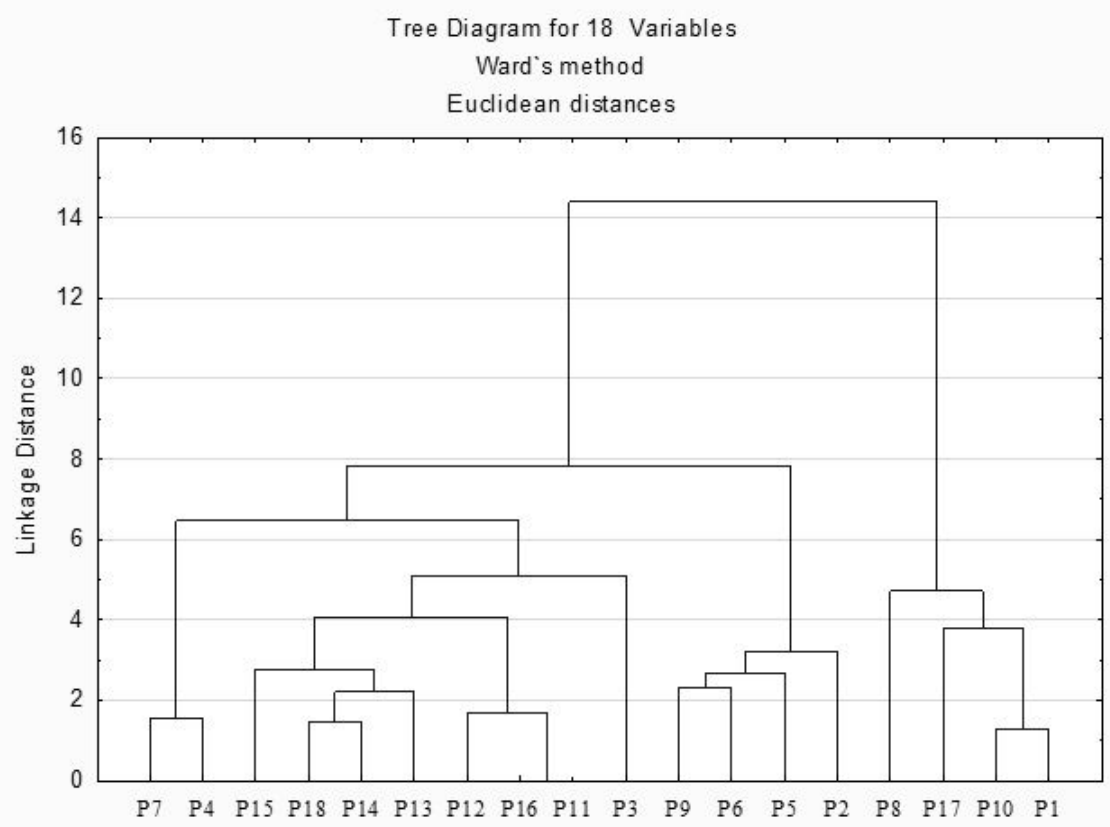

Figure 2. Clusters of morphometry and plumage parameters of Motacilla citreola obtained with Ward method

After clustering of the results of the pairwise correlation analysis of morphometry and plumage features by Ward three clusters of samples of yellow wagtail $M$. flava associated with presence of the specimens of subspecies $M$. $f$. flava and M. f. thunbergi in the samples were identified. One specimen turned out to be a hybrid, as well as three 
clusters of samples of citrine wagtail $M$. citreola associated with the presence of the specimens of subspecies $M$. c. citreola and M. c. werae in the samples. Two specimens were hybrid (Figure 3, 4).

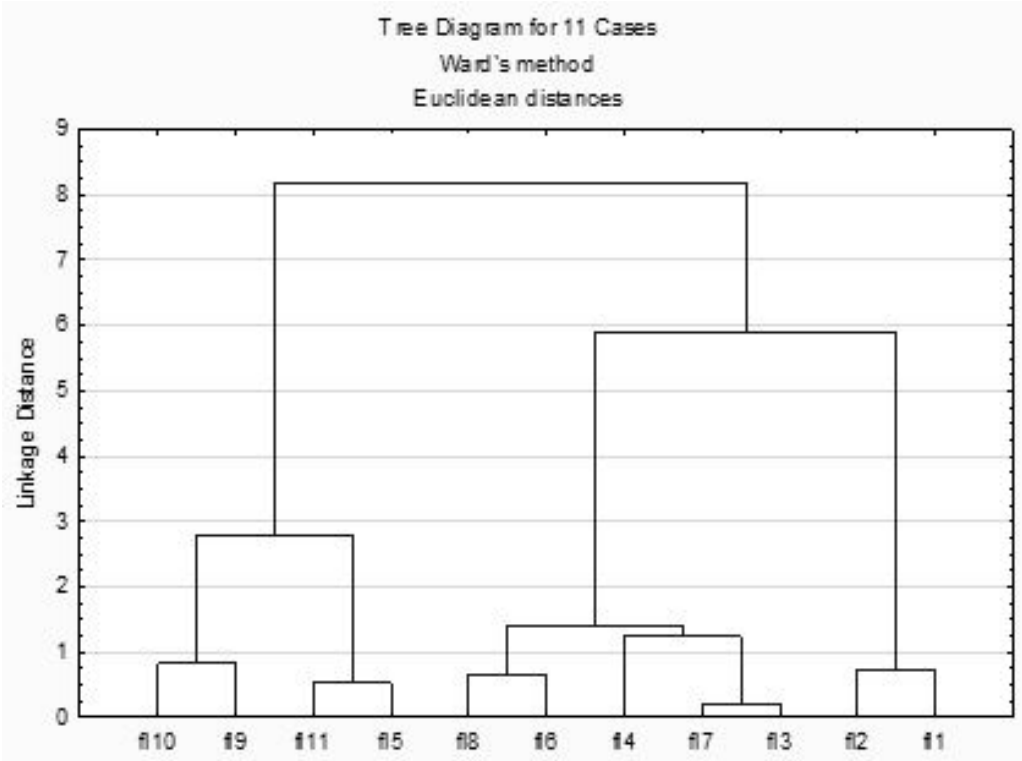

Figure 3. Clusters of Motacilla flava in the space of informative parameters obtained by Ward method

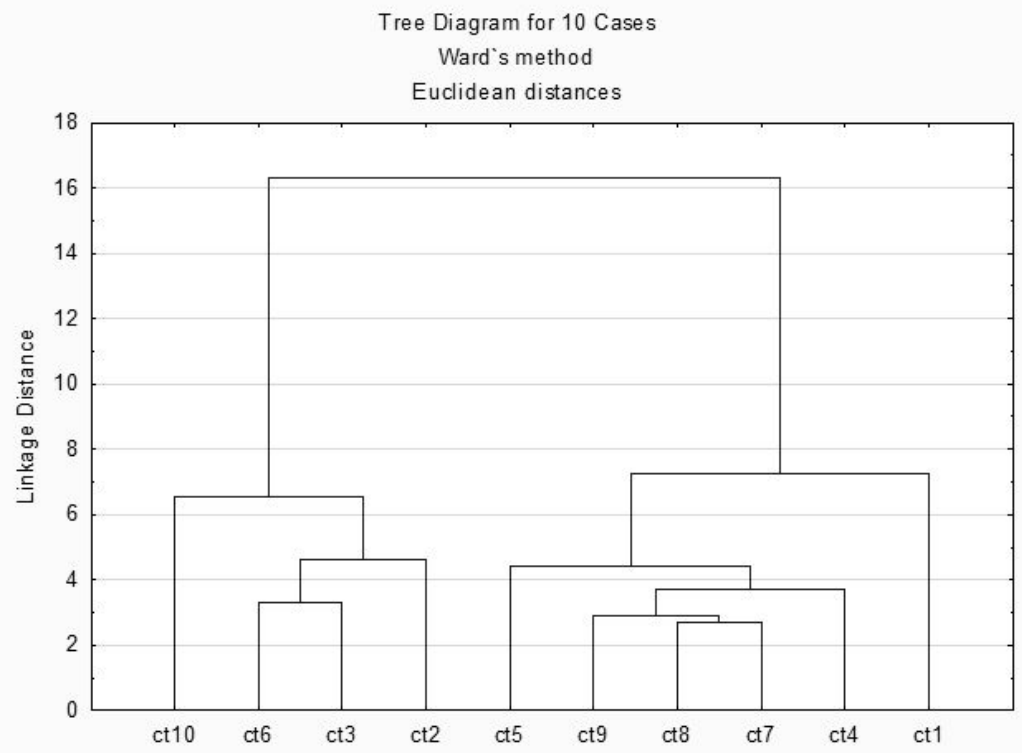

Figure 4. Clusters of Motacilla citreola in the space of informative parameters obtained by Ward method

When sequencing the amplified DNA fragments of $M$. flava blood samples sequences of the gene cytochrome c oxidase I were obtained. Phylogenetic trees were constructed using Neighbor-joining method. The sequences of the gene of interest turned out to be different in all dry samples taken from individual birds suggesting genetic heterogeneity of $M$. flava and $M$. citreola populations of wagtails in the studied area. The sequences were aligned using ClustalW2 software, then phylogenetic trees for all specimens of $M$. flava and $M$. citreola populations were constructed using JalView software and genetic distances were defined (Table 5 and 6; Figure 5, 6). 
Table 5. Genetic distances between studied specimens of Motacilla flava samples in Middle Volga populations obtained with MEGA 4 software

\begin{tabular}{lcccccccccccc}
\hline $\begin{array}{l}\text { Number of } \\
\text { the ring }\end{array}$ & XH & XH & XH & XH & XH & XH & XH & XH & XH & XH & XH \\
\hline XH 51067 & - & 0.009 & 0.009 & 0.009 & 0.009 & 0.009 & 0.009 & 0.003 & 0.009 & 0.009 & 0.014 \\
XH 51060 & 0.009 & - & 0.000 & 0.000 & 0.000 & 0.000 & 0.000 & 0.006 & 0.000 & 0.000 & 0.006 \\
XH 51058 & 0.009 & 0.000 & - & 0.000 & 0.000 & 0.000 & 0.000 & 0.006 & 0.000 & 0.000 & 0.006 \\
XH 51053 & 0.009 & 0.000 & 0.000 & - & 0.000 & 0.000 & 0.000 & 0.006 & 0.000 & 0.000 & 0.006 \\
XH 51052 & 0.009 & 0.000 & 0.000 & 0.000 & - & 0.000 & 0.000 & 0.006 & 0.000 & 0.000 & 0.006 \\
XH 51051 & 0.009 & 0.000 & 0.000 & 0.000 & 0.000 & - & 0.000 & 0.006 & 0.000 & 0.000 & 0.006 \\
XH 51048 & 0.009 & 0.000 & 0.000 & 0.000 & 0.000 & 0.000 & - & 0.006 & 0.000 & 0.000 & 0.006 \\
XH 51046 & 0.003 & 0.006 & 0.006 & 0.006 & 0.006 & 0.006 & 0.006 & - & 0.006 & 0.006 & 0.011 \\
XH 51039 & 0.009 & 0.000 & 0.000 & 0.000 & 0.000 & 0.000 & 0.000 & 0.006 & - & 0.000 & 0.006 \\
XH 51036 & 0.009 & 0.000 & 0.000 & 0.000 & 0.000 & 0.000 & 0.000 & 0.006 & 0.000 & - & 0.006 \\
XH 50736 & 0.014 & 0.006 & 0.006 & 0.006 & 0.006 & 0.006 & 0.006 & 0.011 & 0.006 & 0.006 & - \\
\hline
\end{tabular}

Table 6. Genetic distances between studied specimens of Motacilla citreola samples in MiddleVolga populations obtained with MEGA 4 software

\begin{tabular}{llllllllll}
\hline $\begin{array}{l}\text { Number of the } \\
\text { ring }\end{array}$ & XH & XH & XH & XH & XH & XH & XH & XH & XH \\
XH 51047 & 51047 & 51049 & 51041 & 51068 & 51045 & 51042 & 47691 & 51043 & 51044 \\
XH 51049 & - & 0.011 & 0.007 & 0.007 & 0.011 & 0.004 & 0.004 & 0.004 & 0.011 \\
XH 51041 & 0.007 & 0.004 & - & 0.000 & 0.011 & 0.004 & 0.004 & 0.004 & 0.011 \\
XH 51068 & 0.007 & 0.004 & 0.000 & - & 0.011 & 0.004 & 0.004 & 0.004 & 0.011 \\
XH 51045 & 0.011 & 0.015 & 0.011 & 0.011 & - & 0.007 & 0.007 & 0.007 & 0.015 \\
XH 51042 & 0.004 & 0.007 & 0.004 & 0.004 & 0.007 & - & 0.000 & 0.000 & 0.007 \\
XH 47691 & 0.004 & 0.007 & 0.004 & 0.004 & 0.007 & 0.000 & - & 0.000 & 0.007 \\
XH 51043 & 0.004 & 0.007 & 0.004 & 0.004 & 0.007 & 0.000 & 0.000 & - & 0.007 \\
XH 51044 & 0.011 & 0.015 & 0.011 & 0.011 & 0.015 & 0.007 & 0.007 & 0.007 & - \\
\hline
\end{tabular}

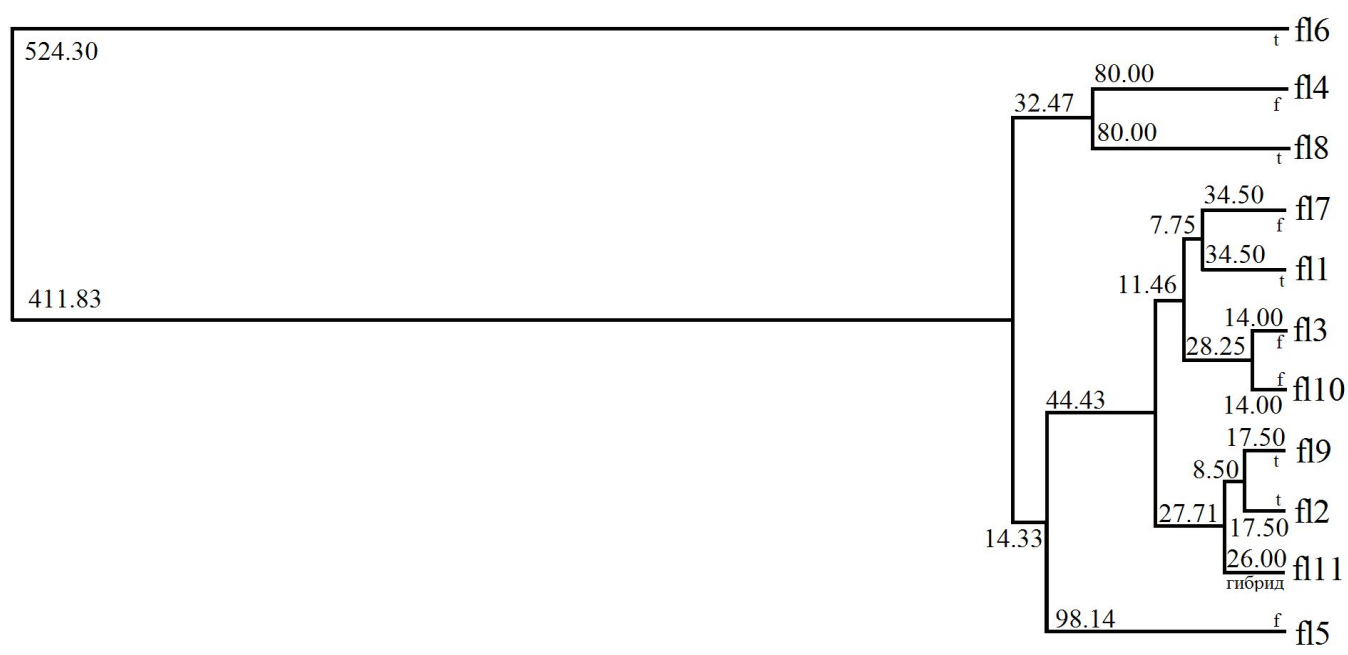

Figure 5. Phylogenetic tree of Motacilla flava specimens constructed on the base of genetic analysis of sequences of the gene cytochrome c oxidase I using JalView software, Average Distance method (weighted average) 


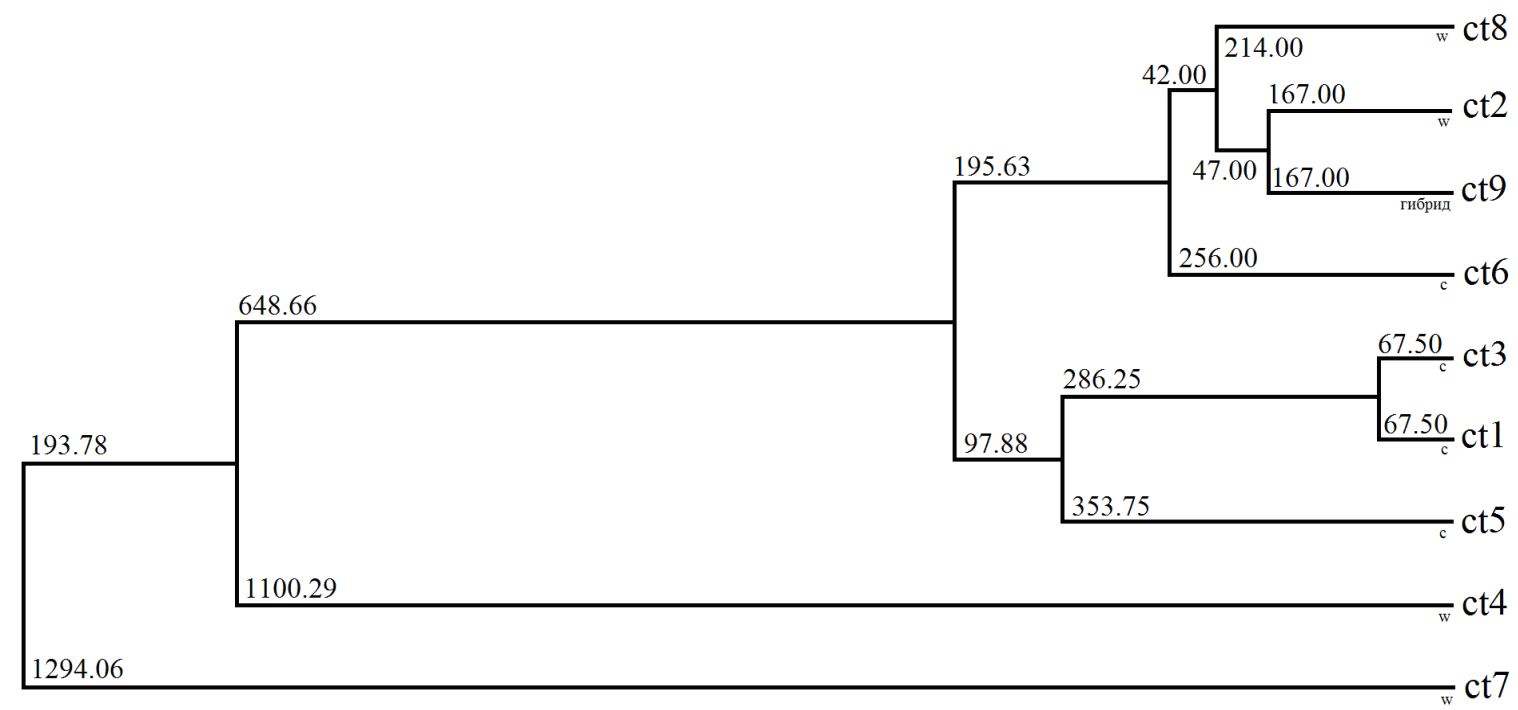

Figure 6. Phylogenetic tree of Motacilla citreola specimens constructed on the base of genetic analysis of sequences of the gene cytochrome c oxidase I using JalView software, Average Distance method (weighted average)

\section{Discussion}

During phylogeographic analysis of nucleotide sequences of mitochondrial genes in the Middle Volga populations of traditionally recognized species $M$. flava and $M$. citreola the lines common in the European part of Russia and neighboring countries which respectively meet subspecies $M$. f. flava and $M$. f. thunbergi were revealed; the third line is related to presence of the hybrid specimens in the studied populations. Within the species $M$. citreola three lines were also revealed corresponding $M . c$. citreola and $M . c$. werae subspecies. This third line also corresponds to the presence of hybrid specimens in the Middle Volga populations.

\subsection{Phenotypic and genotypic structure of M. flava populations}

Coloration of the "whiskers" (the most informative indication of plumage color) of the specimens of the $M$. flava subspecies varies from yellow to white in the M.f. thunbergi and from gray to yellow-gray in M.f.flava. According to the genetic research five specimens belong to the $M$. $f$. thunbergi subspecies, 6 - to the $M$. f. flava subspecies (5 thunbergi: 6 flava). In the subspecies M.f. thunbergi phenotypic splitting of "whiskers" plumage had the next form: 1 specimen with yellow "whiskers", 2 specimens with half yellow (half white) "whiskers", 2 specimens with white "whiskers". In the subspecies $M . f$. thunbergi phenotypic splitting of "whiskers" plumage had the next form: 1 specimen with gray yellow "whiskers", 2 specimens with gray "whiskers", 1specimen with half white "whiskers" and 2 specimens with white "whiskers". The overall ratio of phenotypes: - 4 white : 3 half yellow (half white): 2 gray : 1 yellow : 1 gray yellow. Probably, this feature can be inherited by the complementarity or incomplete dominance type.

\subsection{Phenotypic and genotypic structure of M. citreola populations}

The presence of "necklace" (the most informative indication of plumage color) in the M. citreola subspecies varies discretely - specimens of the $M$. c. citreola subspecies have necklace and specimens of the M. $c$. werae subspecies have not. Hybrid specimens also have two similar kinds of phenotype by the presence of "necklace". In the performed molecular genetic study of $10 \mathrm{M}$. citreola specimens 4 specimens belonged to the subspecies $M$. $c$. werae, 4 specimens - to subspecies $M$. c. citreola ( 4 werae: 4 citreola), two specimens turned out to be hybrid. In the subspecies M. c. citreola phenotypic splitting of "necklace" had the next form: 2 specimens had a necklace and 2 specimens hadn't. 4 specimens of the subspecies $M$. c. werae had no "necklace". Phenotype ratio for a given parameter was 7: 3 (2:1). Probably, this feature can be inherited as following test cross: allele Aa (no "necklace") - M. c. werae genotype, AA allele ("necklace") - M. c. citreola genotype. Then, when crossed the next splitting will be: 2 Aa (werae): 1 aa (citreola).

Nevertheless, these subspecies rather differ in some peculiarities of their biology, ecology and morphology. So we can consider these forms at the level of species as evidenced by the comparative analysis of mitochondrial DNA. Defined genotype and phenotype ratios of the area space can mark the area of hybridization of studied phenotypes. 


\section{Conclusions}

Genetic structure of Middle Volga populations of "yellow" wagtails M. flava and M. citreola is heterogeneous. Subpopulations with prevailing subspecies M. f. flava and M. f. thunbergi or subspecies M. c. citreola and M. c. werae were revealed. Also hybrid specimens were observed in these populations. At that $M$. f. flava and M. $f$. thunbergi as well as M. c. citreola and M. c. werae are well genetically differed with maximum genetic distances equal to 524,30 and $1100,29,1294,06$ respectively. These distances correspond to subspecies level of differences in M. flava and to species level in M. citreola. Themost in formative parameters of morphometry and plumage color were revealed: tarsus length, body length and "whisker" coloration in M. flavaand also tarsus length, body length and necklace besides in M. citreola.

Subspecies forms of M. flava viz. M. f. flava, M. f. thunbergi are included in the Western complex of $M$. flava forms; subspecies forms of M. citreola viz. M. c. citreola, M. c. werae form a separate genetic branch of the polytypical group M. flavas.1. [11]. In the North-Western and Northern Europe form M. f. thunbergiis common where mixed populations of M.f. flava and M.f. thunbergi nest. Throughout its area males of M.f. thunbergiform live sympatrically with white-brow form of $M$. f. flava generating all variants of transitions between these forms by hybridization. Spectrum of autogenetic processes in M. flava and M. citreola populations in area space in wide sympatric conditions reflects the mechanisms of reproductive isolation of forms of species and subspecies rank. It is the result of microevolution of $M$. flava polytypic complex.

Thus, the possibility of genetic separation between sympatric breeding populations of yellow wagtail Motacilla flava and citrine wagtail Motacilla citreola in the Middle Volga was revealed.

Phylogeographical analysis of the nucleotide sequences of the mitochondrial gene cytochrome oxidase I in studied populations of "yellow" wagtails revealed the existence of separate lines common in the European part of Russia and neighboring countries including subspecies M. f. flava, M. f. thunbergi and M. c. citreola, M. c. werae respectively.

So forms M. c. citreola and M. c. werae need assigning them the status of species due to significant genetic distances.

Despite widespread sympatry in nesting habitats there is a selective mating between males and females of each studied species that prevents free crossing and supports isolating mechanisms in the populations.

\section{Acknowledgements}

The authors express their sincere gratitude to the staff of Eco-Center "Dodo" A.I. Matsynya and E.L. Matsynya for the opportunity to work at the bird ringing station and practical help; to Ph.D., the Head of Department of Informatics of UISPU V.G. Shubovich for assistance with the Statistica 10 software. This study was performed under the support of the federal target program of Ministry of Education of the Russian Federation "Goszadanie 2015/391 for 2015", project number 2607.

\section{References}

Artemieva, E. A., \& Muraviev, I. V. (2012a). By the history of studying of the variability of plumage color of "yellow" wagtails (Passeriformes, Motacillidae, Motacillinae): from NA Zarudny to our days. (K istorii izucheniya izmenchivosti okraski opereniya "zhyoltykh" tryasoguzok (Passeriformes, Motacillidae, Motacillinae) ot N.A. Zarudnogo do nashykh dney). Proceedings of the international conference "Terrestrial vertebrates of arid ecosystems", devoted to the memory of NA Zarudny (October 24-27; 2012; Tashkent). Tashkent: Chinor ENK, 24-34.

Artemieva, E. A., \& Muraviev, I. V. (2012b). Sympatry of "yellow" wagtails (Passeriformes, Motacillidae, Motacillinae): geography, ecology, evolution. (Sympatriya "zhyoltykh" tryasoguzok (Passeriformes, Motacillidae, Motacillinae): geographiya, ecologiya, evolutsiya). Parts 1, 2. M.: Flinta-Nauka, 2012b. 152 p. $200 \mathrm{p}$.

Babenko, V. G. (1981). Spatial and reproductive relations between two forms of yellow wagtails (O prostranstvennykh I reproductivnykh otnosheniyakh dvukh form zhyoltykh tryasoguzok). Biologicheskie nauki, 3(207), 42-45.

Bakhtadze, G. B. (1987). The distribution of pigment in the feathers of yellow wagtails (Motacilla flava L., M. feldegg Mich., M. lutea Gmel.) and their plumage color (Raspredelenie pigmentov v peryakh zhyoltykh tryasoguzok (Motacilla flava L., M. feldegg Mich., M. lutea Gmel.) I okraska ikh opereniya). Proceedings of the North-Caucasian scientific Center of Higher School. Series of nature sciences. Roston-na-Donu, 1987. 1-5. Deposited in VINITI 18. 08 1987, № 1920. 
Beregovoy, V. E. (1970). Phenotypical analysis of head colorin the contact area of yellow and gray forms (Phenoanaliz okraski golovy zhyoltoi tryasoguzki $\mathrm{v}$ zone kontakta serogolovoi I zheltogolovoi form). Ecologiya, 6, 102-103.

Gladkov, N. A. (1954). The birds of the Soviet Union (Ptitsy Sovetskogo Soyuza). M.: Sovetskaya nauka, 5, 594 690.

Grichik, V. V. (1992). Phenogeography of yellow wagtails' polymorphism due to problems of systematics and genesis of the"Motacilla flava"complex (Phenogeographiya polimorphisma zhyoltykh tryasoguzok v svyazi s problemami systematiki I genezisa kompleksa "Motacilla flava"). Abstract of thesis in biology, Kiyev, 116.

Zarudny, N. A. (1891). About hybrids between Budytes flava L. and Budytes campestris Pall. (O gibridakh mezhdu Budytes flava L. I Budytes campestris Pall.) Proceedings of the St. Petersburg Naturalists' Society. Department of Zoology and Physiology, 22(1), 27-38.

Muraviev, I. V., Artemieva, E. A., \& Beme, I. R. (2014). Geographical distribution, nesting habitats and abundance of citrine wagtail Motacilla citreola Pallas, 1776 (Passeriformes, Motacillidae) in the Middle Volga region (Geographicheskoe rasprostranenie, biotopy gnezdovaniya I chislennost' zheltogolovoi tryasoguzki Motacilla citreola Pallas, 1776 (Passeriformes, Motacillidae) v srednem Povolzhie) // Vestnik Moskovskogo Universiteta, ser. 16. Biologiya. Fauna, Flora, 3, 52-58.

Portenko, L. A. (1960). Birds of the USSR (Ptitsy SSSR). M.- L.: AN SSSR, Part 4 (416 p).

Red'kin, Y. A. (2001a). Juvenile plumage colouration of some forms of yellow wagtails' group Motacilla flava sensu lato (Okraska opereniya nekotorykh form gruppy zhyoltykh tryasoguzok Motacilla flava sensu lato v yuvenil'nom naryade). Russkiy Ornitologicheskiy Jurnal, Express edition, 128, 3-27.

Red'kin, Y. A. (2001b). Taxonomic relations of the forms in evolutionally young bird complexes on the example of the genus Motacilla L., 1785: taxonomic revision of subgenus Budytes (Taksonomicheskie otnosheniya form v evolutsionno molodykh kompleksakh ptits na primere roda Motacilla L., 1785). Abstract of thesis in biology (19 p).

Ryabitsev, V. K. (2001). Birds of Urals, Suburals and Western Siberia(Guide) (Ptitsy Urala, Priuralia I Zapadnoy Sibiri). Ekaterinburg: Publishing of the Ural University, 1-608.

Sotnikov, V. N. (2006). Birds of the Kirov region and adjacent territories. Passerine. V. 2. Part 1. Kirov: OOO "Triada+"Ltd., 2006. 448 p.

Stepanyan, L. S. (1990). Summary of the ornithological fauna of the USSR. M.: Nauka, 366 p.

Vili, N., Chavko, J., Szabó, K., Kovács, S., Hornung, E., Kalmár, L., \& Horváth, M. (2009). Genetic structure of the Imperial Eagle (Aquila heliaca) population in Slovakia. Slovak Rapt J., 3, 21-28.

Artemieva, E. A., Muraviev, I. V., \& Beme, I. R. (2013). Yellow Wagtail Motacilla flava Linnaeus, 1758 (Passeriformes, Motacillidae, Motacillinae), in the Middle Volga Region: Geographical Distribution, Nesting Biotopes, and Numbers. Moscow Univ. Biol. Sci. Bull., 68(3), 131-142.

Cramp, S. (1998). The Birds of the Western Palaearctic (pp. 1-1063). OxfordUniv. Press.

Pavlova, A., Zink, R., Drovetski, S. V., Red'kin, Y., \& Rohwer, S. A. (2003). Phylogeographic patterns in Motacilla flava and Motacilla citreola: species limits and populations history. Auk., 120(3), 744-758.

\section{Copyrights}

Copyright for this article is retained by the author(s), with first publication rights granted to the journal.

This is an open-access article distributed under the terms and conditions of the Creative Commons Attribution license (http://creativecommons.org/licenses/by/3.0/). 